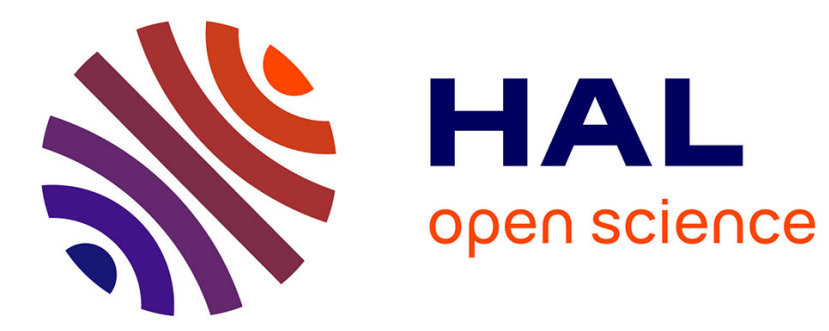

\title{
Les techniques audio-visuelles dans l'enseignement forestier : l'expérience de l'Ecole forestière de Corvallis (Oregon)
}

Michel Thevenon

\section{- To cite this version:}

Michel Thevenon. Les techniques audio-visuelles dans l'enseignement forestier: l'expérience de l'Ecole forestière de Corvallis (Oregon). Revue forestière française, 1971, 23 (5), pp.505-510. $10.4267 / 2042 / 20521$. hal-03395057

\section{HAL Id: hal-03395057 https://hal.science/hal-03395057}

Submitted on 22 Oct 2021

HAL is a multi-disciplinary open access archive for the deposit and dissemination of scientific research documents, whether they are published or not. The documents may come from teaching and research institutions in France or abroad, or from public or private research centers.
L'archive ouverte pluridisciplinaire HAL, est destinée au dépôt et à la diffusion de documents scientifiques de niveau recherche, publiés ou non, émanant des établissements d'enseignement et de recherche français ou étrangers, des laboratoires publics ou privés. 


\title{
LES TECHNIQUES AUDIO-VISUELLES DANS L'ENSEIGNEMENT FORESTIER
} L'eXPÉrience de l'École forestière de corvallis (OREGon)

\author{
M.-J. THEVENON
}

Class. Oxford 945.31 (73)

Beaucoup d'efforts de recherche technique ont été consacrés à l'amélioration de la productivité de nos ressources forestières dans la perspective de pourvoir à nos besoins de consommation et de récréation. Cependant, l'enseignement forestier, qui est l'investissement intellectuel nécessaire au développement de nos industries de la forêt et du bois, ne tire pas toujours profit des nouveaux outils que nous offre la technologie moderne pour améliorer la formation des techniciens et des ingénieurs. L'exemple du Département audio-visuel de l'Ecole forestière de Corvallis (Oregon, Etats-Unis) peut être utile pour se rendre compte du potentiel éducatif que représentent les techniques audio-visuelles. Le but de cet article est donc de présenter les fonctions de ce Département, les moyens d'acquisition des matériaux audio-visuels et les problèmes que pose la création d'un tel département dans un cadre traditionnel d'enseignement.

\section{L'ECOLE FORESTIERE DE CORVALLIS ET SON DEPARTEMENT AUDIO-VISUEL}

L'Oregon State University, où se trouve l'Ecole forestière, est située à Corvallis à 80 kilomètres de la Côte pacifique des Etats-Unis et au cœur même de la célèbre région forestière dont le "Douglas" a fait la réputation. 450 élèves suivent les cours de l'Ecole forestière. Chaque année, 80 d'entre eux reçoivent le diplôme de « Bachelor of Science " après quatre ans d'université (l'entrée à l'université se faisant à l'âge de 18 ans), 15 le diplôme de "Master of Science " après deux ans d'études supplémentaires, et trois ou quatre le doctorat (Ph.D.).

Les spécialités enseignées correspondent à presque tout l'éventail des sciences biologiques et forestiéres ainsi que du génie forestier. II existe aussi de nombreux cours relatifs aux propriétés du bois et aux industries du bois et des pâtes et papiers. Près d'un tiers des élèves qui préparent le "Master of Science " suivent cette orientation. Les cours de formation générale, tels que statistique, mathématiques, physique et chimie, sont donnés dans les autres écoles situées dans le campus universitaire. La plupart des professeurs travaillent au Laboratoire de recherche forestiere. Ils peuvent ainsi incorporer dans leurs cours les derniers résultats de leurs recherches.

L'Ecole, au même titre que l'Université, a la responsabilité de fournir à ces nombreux étudiants une bonne éducation, surtout au niveau des premières années d'études, où l'enseignement est particulièrement difficile. A cette fin, la direction de l'Ecole 
a décidé d'améliorer les techniques d'enseignement traditionnelles et de créer un Département audio-visuel de conception nouvelle. Son développement, sous l'initiative de son directeur actuel, le Dr Reichart (1967), a été rendu possible grâce à une donation de la fondation Maud-Hill.

\section{FONCTIONS DU DEPARTEMENT AUDIO-VISUEL}

Le Département a évolué peu à peu en fonction des besoins de l'Ecole forestière et du Laboratoire de recherche forestière. Il est à la fois un centre d'auto-instruction. un service de prêt et un atelier de préparation de matériaux d'enseignement.

\section{Centre d'auto-instruction}

Le Département comprend principalement une photothèque et une magnétothèque. L'étudiant ou le visiteur peut choisir parmi 700 sujets différents, tous relatifs aux sciences et techniques forestières ainsi qu'aux industries du bois et des pâtes et papiers. II y a environ 300 montages sonorisés, chacun étant un ensemble de diapositives commentées par une bande magnétique synchronisée, 200 bandes magnétiques indépendantes, $50 \mathrm{films}, 20$ films de projection sur écran de télévision, et le reste se compose de documents imprimés.

Douze étudiants peuvent travailler individuellement ou en groupe dans des compartiments équipés d'un magnétophone et d'un projecteur de diapositives. Un assistant distribue les matériaux audio-visuels, montages sonorisés relatifs au sujet désiré, et aide, si nécessaire, l'étudiant à se familiariser avec la manipulation des instruments.

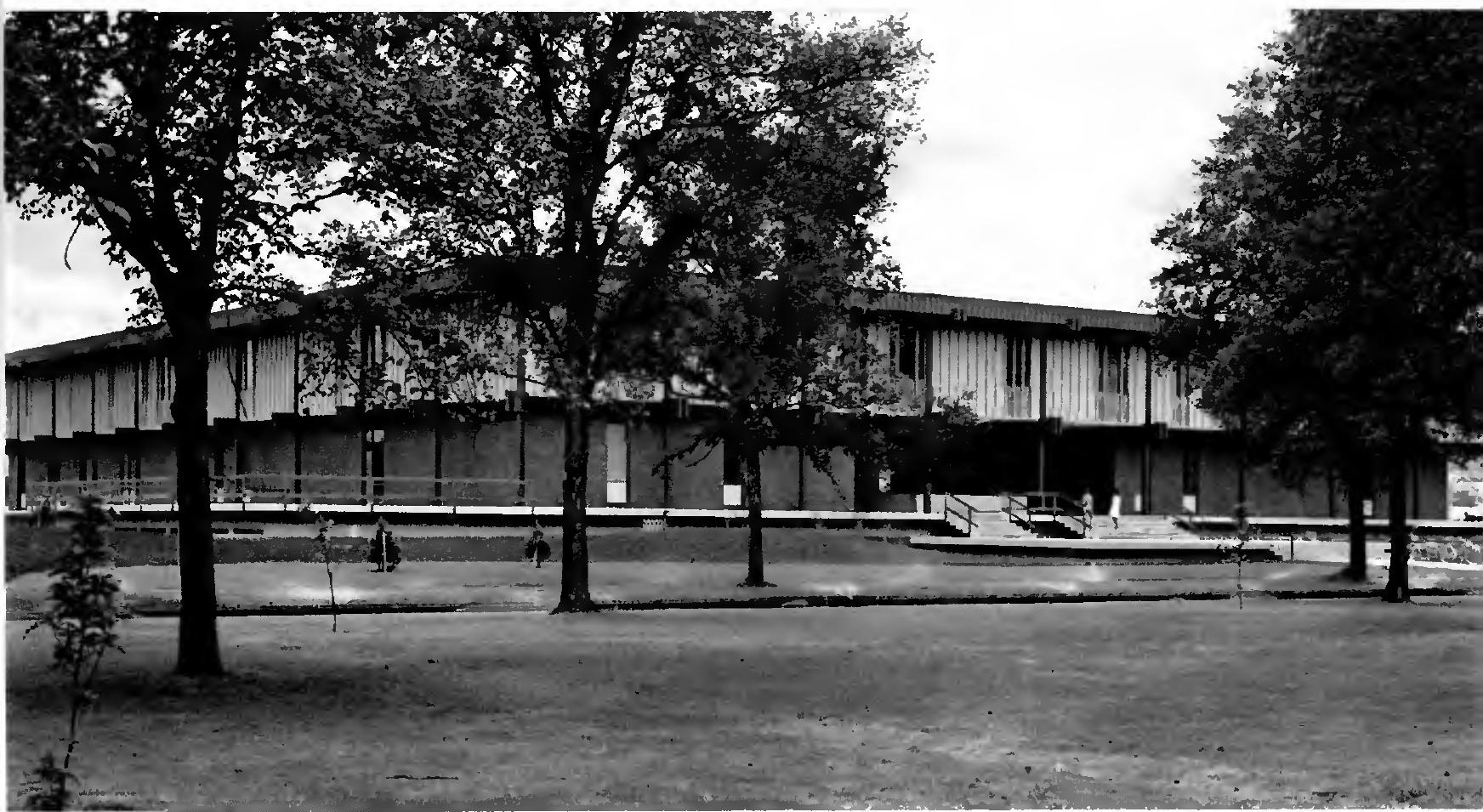


L'élève, en venant au Département, est certain de trouver une documentation récente et de qualité sur les sujets les plus divers tels que, pour en citer quelquesuns : les carrières offertes à la sortie de l'Ecole forestière, le Fortran, la reconnaissance des arbres, la construction de routes, l'utilisation d'explosifs, le débardage par ballon, l'aménagement des forêts, l'étude économique des ressources forestières d'un district de l'Oregon, les inventaires forestiers, la comptabilité forestière, la mycologie forestière, l'utilisation de photographies aériennes pour la lutte contre les incendies en forêt, les adhésifs dans l'industrie du bois, la fabrication du contreplaqué, la visite d'une usine de pâte kraft.

Il apparaît que tous ces matériaux sont une aide précieuse pour les éléves. Plus de 50 étudiants par jour viennent les consulter. Rien ne va à l'encontre de leur utilisation. Ils ne sont pas nécessairement un substitut du professeur, mais un supplément aux cours aidant à la fois le professeur à transmettre le message et les élèves eux-mêmes à mieux le comprendre. L'élève, grâce à la bande magnétique, peut retrouver le message qui dans un cours serait mal pris en note ou bien perdu complètement. II peut s'arrêter, réfléchir et éventuellement revenir en arrière. Le magnétophone a une patience sans fin contrairement, bien souvent, à l'orateur.

De plus, ces matériaux ont un intérêt réel non seulement pour les étudiants qui veulent s'instruire par eux-mêmes ou qui doivent travailler sur un projet indépendant du cours, mais aussi pour les visiteurs qui veulent se documenter sur un sujet précis. La visite du Département apparaît souvent pour le néophyte comme le point de départ, et parfois aussi le point d'aboutissement de ses recherches. En conséquence, il est souvent conseillé de passer au Département audio-visuel avant même de consulter les répertoires de la littérature forestière.

Dans cette perspective un département audio-visuel semble avoir un rôle prometteur non seulement dans la formation régulière des ingénieurs et des techniciens mais aussi dans la formation continue.

\section{Service de prêt}

Le Département prête, aux professeurs et aux élèves, des magnétophones, des projecteurs de film ou de diapositives, des écrans, des omniscopes et des appareils photographiques. En 1970, il a été emprunté en moyenne 70 appareils par mois.

Les magnétophones sont utilisés, la plupart du temps, d'une manière personnelle, par ceux qui désirent se perfectionner en vue d'une conférence ou d'un cours. Beaucoup d'équipement est emprunté ou utilisé sur place par les étudiants. En effet, depuis la création du Département audio-visuel, les professeurs ont tendance à encourager, à la place de devoirs écrits, des présentations orales accompagnèes de diapositives qui sont à préparer par les étudiants eux-mêmes.

Ainsi, un groupe d'élèves a récemment prẻparé, pour un cours de génie papetier, une bande magnétique synchronisée avec une présentation de diapositives portant sur les techniques de fabrication des non-tissés. L'enregistrement et la reproduction des photos à partir de brochures techniques ont été exécutés dans les installations du Département. Ce montage sonorisè, après avoir été présenté avec succès au reste de la classe, a été ajouté à la collection permanente des matériaux audio-visuels. Des rapports de visites ou de stages sont fréquemment présentés de la même manière. Dernièrement, un élève ayant fait un stage dans un chantier d'exploitation forestière où éłait utilisé le ballon a donné au Département un film excellent sur le débardage par ballon, accompagné d'un commentaire enregistré. L'élève bénéficie, dans de tels cas, non seulement de l'équipement du Département, mais aussi des conseils du directeur et de ses assistants en ce qui concerne les prises de vue et la préparation du texte pour l'enregistrement. 


\section{Ateller de préparation de matérlaux d'enseignement}

La plupart des professeurs utilisent des matériaux audio-visuels pour renforcer leur rhétorique personnelle. Le professeur décide ce qu'il veut enseigner ; il appartient alors aux assistants du Département de le soulager dans la préparation des divers matériaux d'enseignement tels que diapositives et tableaux. Le Département prépare par mois plus de 700 photos en y comprenant les photos des publications du Laboratoire de recherche forestière.

De plus, le Département entreprend des travaux plus élaborés, tels que, pour en donner un exemple, l'enregistrement, sur cassettes de magnétophone portatif, des caractéristiques de tous les arbres de l'arboretum de l'Ecole. L'éléve, maintenant, peut écouter ces enregistrements en suivant un parcours prédéterminé le long duquel les arbres et arbustes sont repérables à l'aide d'une carte. Cette forme d'instruction indépendante s'est montrée très efficace et très apprécièe par les élèves.

Le Département a aussi la charge d'assurer les prises de son sur magnétophone des tables rondes et des conférences présentées à l'Ecole par des professeurs éminents. Les enregistrements sont ensuite transcrits en vue de la publication des comptes rendus ou conservés comme documents permanents à la magnétothèque.

\section{MOYENS D'ACQUISITION DES MATERIAUX AUDIO-VISUELS}

Pour constituer la collection de matériaux audio-visuels, trois moyens ont été employés : l'enregistrement des cours de l'Ecole, la préparation spéciale de montages sonorisés et l'achat de matériaux déjà existants.

II est apparu que le meilleur moyen d'obtenir les documents de base était d'enregistrer directement les cours donnés à l'Ecole. Cela n'a cependant pas été facile à mettre en pratique, certains professeurs affirmant que des enregistrements ne serviraient à rien et que les èlèves n'en auraient pas besoin. Néanmoins, d'autres ont coopéré et leurs cours ont été enregistrés. Plusieurs d'entre eux, peu satisfaits des résultats, ont préparé une nouvelle bande magnétique à laquelle ont été jointes des diapositives. Dans des cas plus rares, le professeur a refusé que son enregistrement soit utilisé pour des études auto-didactiques. En réunissant ces bandes magnétiques pour le Département, son directeur a eu la surprise de constater que des professeurs étaient vraiment intéressés par le projet et qu'ils avaient tiré un grand profit des enregistrements. En effet, l'occasion leur avait été fournie de se juger eux-mêmes, de se corriger et de mieux enseigner par la suite.

Un autre moyen a été la préparation de montages sonorisés par le personnel enseignant de l'Ecole. Ainsi, une équipe de quatre professeurs a préparé un cours complet sur la préservation du bois. II est divisé en quatre montages sonorisés, chacun portant sur un sujet particulier : la structure du bois (33 minutes d'enregistrement, 115 diapositives), les principes de séchage (30 minutes, 76 diapositives), la pénétration des liquides dans le bois (20 minutes, 80 diapositives) et les procédés d'imprégnation sous pression (24 minutes, 119 diapositives). Ce cours est présenté de telle manière qu'il peut être utilisé comme moyen d'auto-instruction ou bien être incorporé dans un cours traditionnel.

Etant donné que la préparation et l'organisation d'un montage sonorisé compte pour plus de $95 \%$ de son prix de revient total, les établissements d'enseignement forestier se les vendent. Ainsi, le Département audio-visuel de Corvallis a mis en vente son nouveau cours de préservation du bois pour la somme de 325 dollars. Un cours sur 


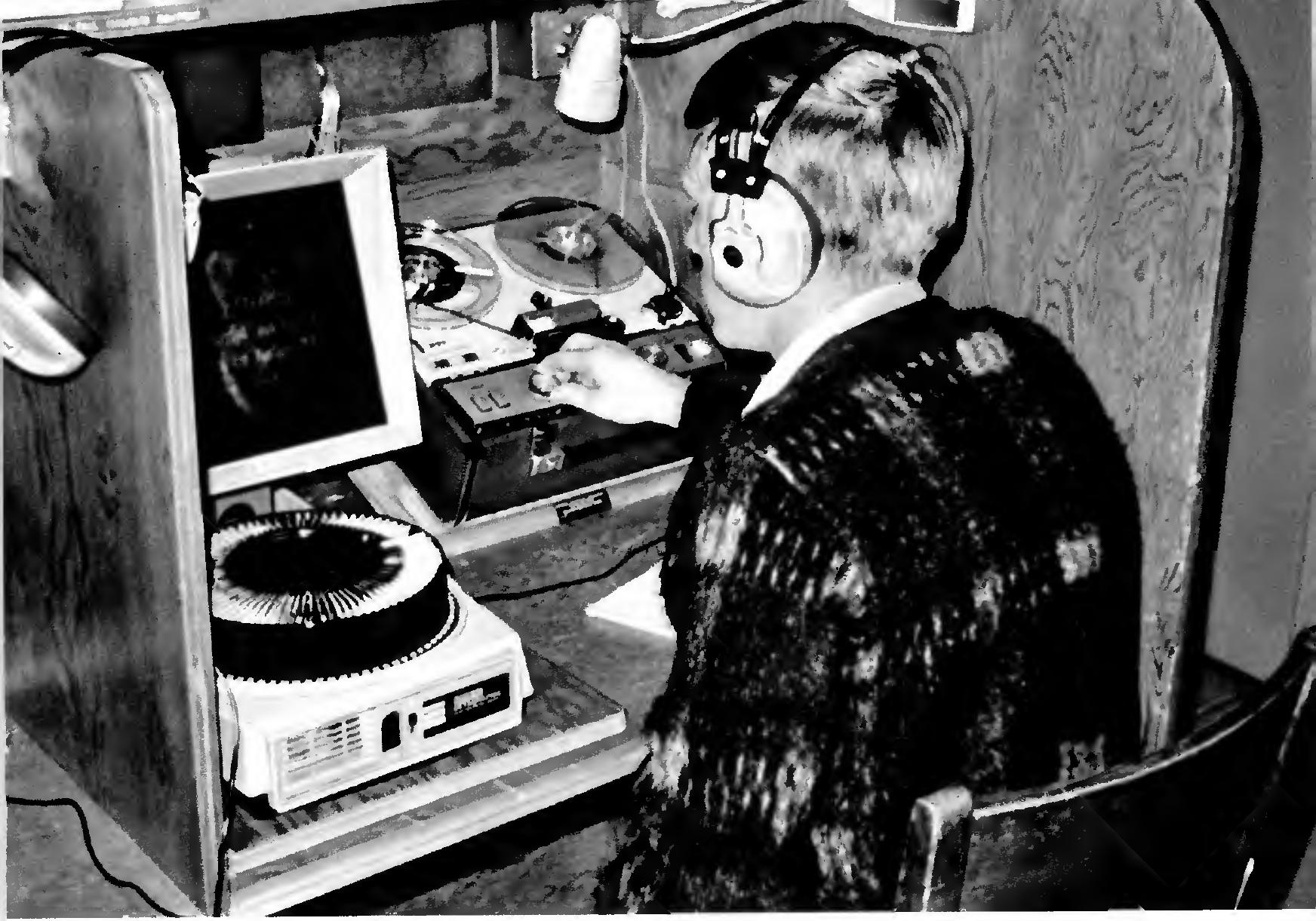

Compartimant d'auto-Instruction ovec magnétophone et projecteur de dlapositivea

Photo THEYENON

le même sujet de 50 minutes et 143 diapositives existe aussi. II est destiné aux sessions de recyclage qul ont lieu dans différentes écoles aux Etats-Unis.

Dans cette perspective, le montage audio-visuel, comme le livre, est un moyen de communication pour diffuser les connaissances. Sa préparation demande beaucoup de recherche, de synthèse et de compréhension de l'audience à laquelle il est destiné. Cet effort d'innovation doit étre reconnu. II est donc apparu nécessaire de protéger ces nouveaux montages sonorisés par des droits d'auteur, ce qui pourrait favoriser la recherche dans le domaine de l'enseignement forestier.

\section{L'AUTO-INSTRUCTION EST-ELLE UN SUBSTITUT DU PROFESSEUR ?}

De nombreuses études ont été faites pour comparer l'efficacité relative de divers procédés d'enseignement (Dubin, p. 35). Les résultats encore très partiels de ces recherches ont établi que l'emploi des systèmes d'auto-instruction permet de communiquer des notions aussi bien que par les formes traditionnelles de l'enseignement. Résultats qui peuvent signifier simplement que les procédés de l'enseignement traditionnel sont aussi peu efficaces que n'importe quels autres.

Cependant, de telles recherches ne tiennent pas compte de la motivation des élèves, des caractéristiques des procédés pédagogiques et des besoins spécifiques de 


\section{M.-J. THEVENON}

chaque établissement d'enseignement. En réalité, l'utilisation des méthodes audio-visuelles dans l'enseignement ne peut être que partielle et ses effets d'ordre essentiellement qualitatlf.

Les méthodes audio-visuelles s'inscrivent dans le mouvement de libération de la fonction enseignante. Elles permettent au professeur de se libérer de certaines tâches secondaires et fastidieuses de présentation et de répétition afin de mieux distribuer ses efforts sur les élèves. Loin de " robotiser " le professeur, la machine vient le " démécaniser ", l'aider à personnaliser son enseignement et à se consacrer plus directement et plus longuement aux tâches nobles de la recherche et du dialogue avec l'étudiant, pour lesquelles il est irremplaçable.

La réaction des étudiants envers les méthodes audio-visuelles est nette : "Ils ne veulent pas réciter comme des singes qui apprennent avec des machines " (Fagan, p. 16). IIs désirent dialoguer avec le professeur, découvrir et discuter avec lui des situations nouvelles. Le rôle du professeur n'est donc pas nécessairement de délivrer des connaissances, mais bien plutôt d'entraîner et de guider les élèves vers la connaissance. II cesse d'être l'oracle et l'origine de tout savoir pour devenir « directeur d'études ou guide d'opinion » (Dieuzeide, p. 115).

De ce point de vue, les méthodes audio-visuelles n'apparaissent certainement pas comme un substitut du professeur. Elles sont des techniques supplémentaires à sa disposition pour découvrir avec l'élève de nouvelles connaissances.

\section{CONCLUSION}

L'exemple du Département audio-visuel de l'Ecole forestière de Corvallis montre que les techniques audio-visuelles peuvent contribuer à accroître l'efficacité de l'enseignement forestier

- en libérant le professeur des tâches fastidieuses et répétitives afin qu'il puisse mieux se consacrer à sa fonction de " directeur d'études "; élèves ;

- en aidant le professeur à transmettre plus efficacement ses connaissances aux

- en favorisant l'innovation dans l'enseignement forestier afin que chacun puisse bénéficier des nouvelles acquisitions de la science et de la technique.

\section{Michel THEVENON}

Assistant de recherches

School of forestry

Oregon State University

CORVALLIS

(Oregon 97.331)

\section{BIBLIOGRAPHIE}

DIEUZEIDE (Henrl). - Les technlques audio-vlsuelles dans l'enseignement. - Paris, Presses universltaires de France, 1965. - $159 \mathrm{p}$.

OUBIN (Robert) et TAVEGGIA (Thomas C.). - The teaching-learning paradox : a comparellve analysls of college teaching methods. - Eugene (Oregon), University of Oregon, Center for the advanced study of educational administration, 1968 . $-78 \mathrm{p}$.

ELLIS (Everett L.). - Educatlon In wood sclence and technology. - Madison (Wisc.), Soclety of Wood Science and Technology, 1964. - $187 \mathrm{p}$.

FAGAN (Brian M.). - The educalion of a professor : the sequel. Educational Televislon, vol. 3, no 1, 1971. Pp. 13-16.

REICHART (Robert R.). - Forestry educalion : a different approech. Journal of Forestry, vol. 65, n 9, 1967. pp. 628-631. 